Research Article

\title{
Frequent Recurrences of Genital Herpes Are Associated with Enhanced Systemic HSV-Specific T Cell Response
}

\author{
Michal Holub $\mathbb{D}^{1},{ }^{1}$ Alžběta Stráníková $\left(\mathbb{D},{ }^{1}\right.$ Pavel Chalupa $\mathbb{D}^{2},{ }^{2}$ Simona Arientová $\left(\mathbb{D},{ }^{1}\right.$ \\ Kateřina Roubalová, ${ }^{3}$ and Ondřej Beran $\mathbb{D}^{1}$ \\ ${ }^{1}$ Department of Infectious Diseases, First Faculty of Medicine, Charles University and Military University Hospital Prague, \\ U Vojenské nemocnice 1200, 16902 Prague 6, Czech Republic \\ ${ }^{2}$ Department of Infectious and Tropical Diseases, First Faculty of Medicine, Charles University and Na Bulovce Hospital, \\ Budinova 2, 18081 Prague 8, Czech Republic \\ ${ }^{3}$ Institute of Haematology and Blood Transfusion, Department of Immunology, U Nemocnice 2094/1, 12820 Prague, \\ Czech Republic
}

Correspondence should be addressed to Ondřej Beran; ondrej.beran@lf1.cuni.cz

Received 3 June 2019; Revised 27 November 2019; Accepted 26 December 2019; Published 25 January 2020

Academic Editor: Leigh Anne Shafer

Copyright (C) 2020 Michal Holub et al. This is an open access article distributed under the Creative Commons Attribution License, which permits unrestricted use, distribution, and reproduction in any medium, provided the original work is properly cited.

Objectives. Genital herpes simplex virus (HSV) infection is controlled by HSV-specific T cells in the genital tract, and the role of systemic T cell responses is not fully understood. Thus, we analysed T cell responses in patients with recurrent genital herpes (GH). Methods. Tcell responses to HSV-1 and HSV-2 native antigens and the expression of HLA-DR and CD38 molecules on circulating CD8+ T cells were analysed in adults with high frequency of GH recurrences (19 patients) and low frequency of GH recurrences (7 patients) and $12 \mathrm{HSV}-2$ seronegative healthy controls. The study utilized the interferon- $\gamma$ Elispot assay for measurement of spot-forming cells (SFC) after ex vivo stimulation with HSV antigens and flow cytometry for analysis of the expression of activation markers in unstimulated T cells. Results. The patients with high frequency of GH recurrences (mean number of recurrences of 13.3 per year) had significantly enhanced HSVspecific T cell responses than the HSV-2 seronegative healthy controls. Moreover, a trend of higher numbers of SFC was observed in these patients when compared with those with low frequency of GH recurrences (mean number of recurrences of 3.3 per year). Additionally, no differences in CD38 and HLA-DR expression on circulating CD8+ T cells were found among the study groups. Conclusions. Frequency of GH recurrences positively correlates with high numbers of systemic HSV-specific T cells.

\section{Introduction}

Genital herpes (GH) represents an important health problem. The disease is caused by herpes simplex virus (HSV) type 1 or type 2 entering the body through the genital tract of a nonimmune person. After the primary HSV infection, the virus stays dormant in the dorsal nerve root ganglia. In some individuals with latent infection, the virus reactivates several times yearly and the reactivation may lead to either recurrent $\mathrm{GH}$ or asymptomatic genital shedding of HSV-2 [1].

The latency of HSV in the dorsal nerve root ganglia is maintained by viral, cell, and immune mechanisms [2]. After the reactivation of virus, its replication is suppressed mostly by HSV-specific immunity. It has been postulated that HSV- specific CD8+ T cells are the most important immune cell types in providing immune surveillance in the tissues because in situ hybridization studies revealed their localization in latently infected ganglia and in the proximity of sensory nerve endings in the skin of genitalia of patients with recurrent GH $[3,4]$. Some studies also suggest a role of HSVspecific CD4+ T cells because genital ulcerations caused by HSV-2 are heavily infiltrated by this cell type [5].

Only scarce data are available on systemic HSV-specific $\mathrm{T}$ cell-mediated responses in the blood of patients with recurrent GH. A study of CD4+ T cell-mediated responses after ex vivo stimulation with HSV antigens did not demonstrate significant differences among patients with recurrent GH- and HSV-seropositive healthy controls [6]. In this 
study, the only significant difference in HSV-specific peripheral CD4+ T cell response was found between recurrent GH and HSV meningitis. Similarly, a study of the activation of circulating CD8+ T cells in HSV-2-infected individuals did not reveal significant changes in patients with recurrent GH [7]. On the other hand, a study of T-cell-mediated responses after primary HSV-2 infection reported persistent HSV-2-specific immune responses in a cohort with nonprimary genital HSV infection [8].

Therefore, we aimed to compare T cell-mediated HSVspecific responses after stimulation with either HSV-1 or HSV-2 native antigens in groups of patients with low and high frequency of GH recurrences and HSV-2 seronegative healthy controls. In addition, we compared the expression of activation markers on circulating CD8+ T cells among the study groups.

\section{Patients and Methods}

2.1. Patients. Twenty-six adults with HSV-2 infection were included in the study. All patients were enrolled at an outpatient clinic for chronic HSV infection, Department of Infectious and Tropical Diseases in the Na Bulovce Hospital, Prague, during the years 2011-2012. They were divided into two groups according to the frequency of $\mathrm{GH}$ recurrences: group 1 with a low frequency of $\mathrm{GH}$ recurrences $(<10 \mathrm{re}-$ currences yearly, mean of 3.3 recurrences per year) and group 2 with high frequency of GH recurrences ( $>10$ recurrences yearly, mean of 13.3 recurrences a year). The etiology of GH was confirmed by the detection of HSV-2 DNA in genital ulcerations or by characteristic clinical findings of recurrent genital lesions responding to acyclovir therapy in a patient with positive anti-HSV-2 serology. The control group consisted of $12 \mathrm{HSV}-2$ seronegative healthy controls without a history of GH. The demographic, clinical, and laboratory data of the enrolled subjects are presented in Table 1. This prospective study was conducted in accordance with the Declaration of Helsinki after obtaining approval from the local ethics committee. A written informed consent was required from the study participants prior their enrolment.

\subsection{Methods}

2.2.1. Serology. Specific antibodies were detected with chemiluminescence immunoassay tests for HSV-1/2 IgM, HSV-1/2 IgG, HSV-1 IgG, and HSV-2 IgG (DiaSorin, Saluggia, Italy) using a Liaison analyser (DiaSorin).

2.2.2. Polymerase Chain Reaction. The specimens were obtained from the genital ulceration using Dacron swab FLOQswabs $^{\mathrm{TM}}$ (Copan, Brescia, Italy) and processed on a Chemagic Prepito ${ }^{\mathrm{TM}}$ automatic analyser (PerkinElmer) using nucleic acid isolation with magnetic beads. The Chemagen Prepito Viral DNA/RNA200 kit was used for nucleic acid isolation. HSV DNA was detected by polymerase chain reaction (PCR) using the EliGene HSV-1 RT and EliGene HSV-2 RT tests (Elisabeth Pharmacon, Brno, Czech
Republic) and an EliGene analyser ABI $7300^{\mathrm{TM}}$ (Applied Biosystems Inc, Foster City, CA, USA).

2.2.3. Herpes Simplex Virus Native Antigens. HSV-1-CI native and HSV-2-CI native (Vidia, Vestec, Czech Republic) antigens were prepared as lysates from VERO cells infected with HSV-1 or HSV-2 (strain Praha and strain 910, respectively). When approximately $90 \%$ of the infected cells displayed a cytopathic effect, the cultures were harvested, washed with phosphate-buffered saline (PBS), and extracted with extraction buffer $(0.01 \mathrm{M}$ glycine, $0.1 \mathrm{M} \mathrm{NaCl}, \mathrm{pH} 7.2$, supplemented with the Protease Inhibitor Cocktail, Roche) for 2 hours at $37^{\circ} \mathrm{C}$. Subsequently, the cell debris was diminished by centrifugation for $30 \mathrm{~min}$ at $1900 \mathrm{rcf}$, and the supernatant was lyophilized.

2.2.4. Flow Cytometry. CD8+ T cell expression of CD38 and HLA-DR was measured in peripheral blood mononuclear cells (PMBC) using four-color flow cytometry and monoclonal antibodies against CD3, CD8, CD38, and HLA-DR molecules (BD Biosciences, San Jose, CA, USA). The data were analysed with BD FACSCanto $\mathrm{II}^{\mathrm{TM}}$ and BD FACSDiva ${ }^{\mathrm{TM}}$ software (BD Biosciences).

2.2.5. Elispot Assays. The interferon (IFN)- $\gamma$ Elispot assay was performed from cryopreserved PBMC, as previously described [9] with modifications. Wells were precoated using Capture Antibody Elispot Development Module Human IFN- $\gamma$ (R\&D Systems, Minneapolis, MN, USA). The cells were plated at $1 \times 10^{5} \mathrm{PBMC}$ per well in duplicate. HSV1 and/or HSV-2 native antigens were added to the wells at a final concentration of $5 \mu \mathrm{g} / \mathrm{mL}$. Wells containing phorbol myristate acetate (PMA, $100 \mathrm{ng} / \mathrm{mL})+$ ionomycin $(1 \mu \mathrm{g} / \mathrm{mL})$ or CEF peptide mix of 9 mer-11 mer peptides from cytomegalovirus, Epstein-Barr virus, and influenza proteins $(1.5 \mu \mathrm{g} / \mathrm{mL})$ served as positive controls. Wells containing RPMI served as negative controls. CD4 and CD8 T cell antigen-specific responses were expressed as spot-forming cells (SFC) per $10^{6}$ PBMC after subtraction of the mean patient background.

2.2.6. Ethics Approval. The study was approved by the Ethics Committee of $\mathrm{Na}$ Bulovce Hospital (reference number 14.2.2011/5467/EK-Z).

2.2.7. Statistical Analysis. Statistical differences among group 1, group 2, and the healthy controls were tested with the Kruskal-Wallis test (a nonparametric version of classical one-way ANOVA) using SigmaStat ${ }^{\circledR}$ software version 3 (Jandell Scientific, San Rafael, CA, USA).

\section{Results}

The results of laboratory analyses and the comparison among the groups are shown in Figure 1 and Table 2. HSVspecific T cell responses were significantly higher in patients with high frequency of GH recurrences (140 and 180 SFC for 
TABLE 1: Demographic, clinical, and laboratory data of patients with genital herpes and HSV-2 seronegative healthy controls.

\begin{tabular}{lccc}
\hline Parameter & Group 1 $(n=7)$ & Group 2 $(n=19)$ & Controls $(n=12)$ \\
\hline Sex (male/female) & $0 / 7$ & $7 / 12$ & $4 / 8$ \\
Age (mean, range) & $48.7,21-73$ & $39.2,27-60$ & $35.8,26-61$ \\
Recurrences (mean, range) & $3.3,2-6$ & $13.3,10-24$ & - \\
Detection of HSV DNA in genital swab (+/0) & $4 / 3$ & $14 / 5$ & - \\
Presence of anti-HSV-2 IgG (+/-) & $7 / 0$ & $17 / 2$ & $0 / 12$ \\
Presence of anti-HSV-1 IgG (+/-) & $5 / 2$ & 6 & $8 / 4$ \\
Suppressive therapy (valaciclovir) & 1 & 13 & - \\
Episodicall treatment (valaciclovir or acyclovir) & 4 & - & - \\
Without treatment & 2 & 12 \\
\hline
\end{tabular}

Group 1, patients with low frequency of GH recurrences ( $<10$ recurrences/year); Group 2, patients high frequency of GH recurrences ( $>10$ recurrences/year); + , positive; -, negative.

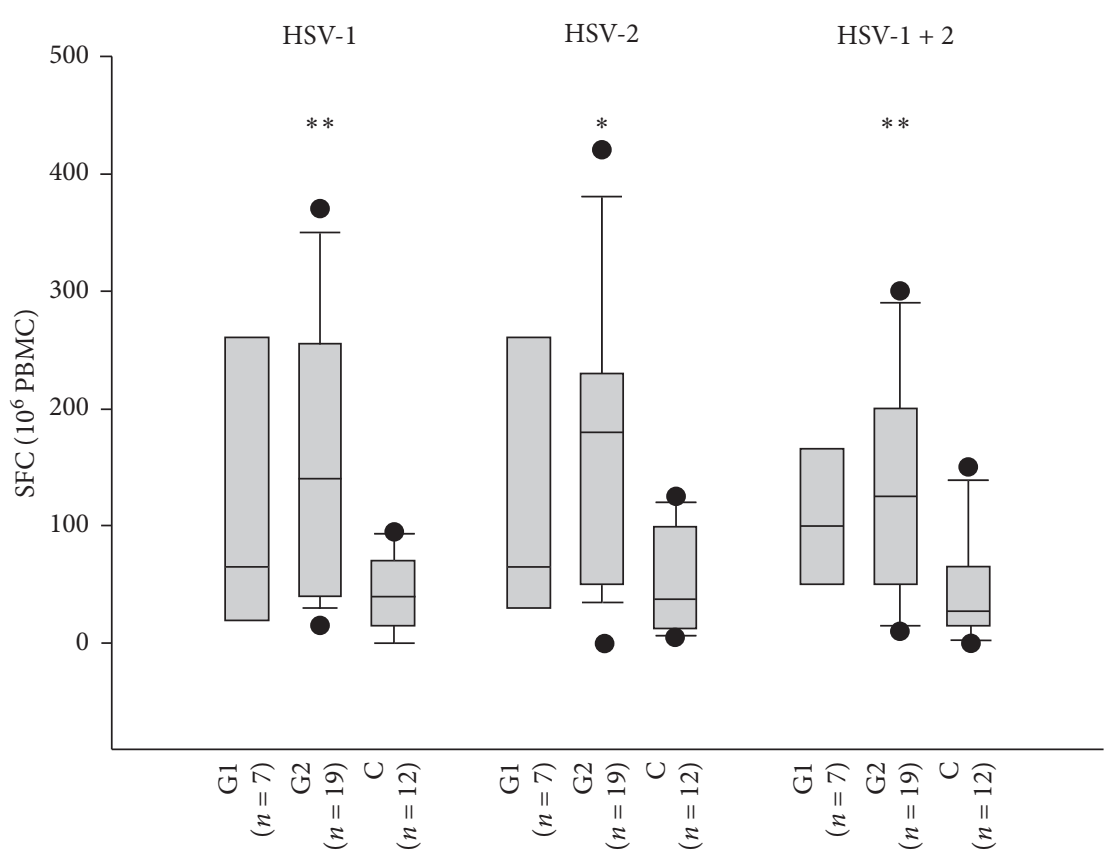

FIGURE 1: The comparison of HSV-1 and HSV-2-specific interferon- $\gamma$ production measured by Elispot in patients with genital herpes and HSV-2 seronegative healthy controls (C). Patient groups: group 1 (G1), patients with $<10$ genital herpes recurrences per a year; group 2 (G2), patients with $>10$ genital herpes recurrences per a year. Interferon- $\gamma$ production is reported as spot-forming cells (SFC) $/ 10^{6}$ peripheral blood mononuclear cells (PBMC). The 25th and 75th percentiles define boxes, and the median values are indicated. The Kruskal-Wallis test was used to detect significant differences among the groups indicated above the boxes versus controls; ${ }^{*} p<0.05,{ }^{* *} p<0.01$.

TABLE 2: Comparison of immunological parameters in patients with genital herpes and HSV-2 seronegative healthy controls.

\begin{tabular}{|c|c|c|c|c|}
\hline Parameter & Group $1(n=7)$ & Group $2(n=19)$ & Controls $(n=12)$ & $p$ value \\
\hline CD8+CD38+T cells (\%; range) & $9.3 ; 8.7-9.9$ & $8.0 ; 6.9-9.9$ & $9.1 ; 6.4-1.1$ & NS \\
\hline CD8+HLA-DR+T cells (\%; range) & $2.6 ; 2.3-1.2$ & $6.3 ; 5.0-8.1$ & $7.0 ; 4.0-1.0$ & NS \\
\hline CD8+CD38+HLA-DR+T cells (\%; range) & $1.4 ; 1.2-5.0$ & $2.3 ; 1.3-2.7$ & $1.9 ; 1.1-4.3$ & NS \\
\hline IFN- $\gamma+$ T cells (SFC, HSV -1 stimulation) & $65 ; 29-224$ & $140 ; 48-249^{*}$ & $40 ; 15-70$ & $<0.01$ \\
\hline IFN- $\gamma+$ T cells (SFC, HSV-2 stimulation) & $65 ; 34-243$ & $180 ; 56-228^{*}$ & $37 ; 15-98$ & $<0.05$ \\
\hline IFN $-\gamma+$ T cells (SFC, HSV $-1+2$ stimulation) & $100 ; 54-150$ & $125 ; 54-199^{*}$ & $27 ; 15-65$ & $<0.01$ \\
\hline
\end{tabular}

Group 1, patients with low frequency of GH recurrences ( $<10$ recurrences/year); Group 2, patients with high frequency of GH recurrences $(>1024$ recurrences/year); SFC, spot-forming cells $/ 10^{6}$ peripheral blood mononuclear cells (mean; range); * significant difference from controls; NS, nonsignificant.

HSV-1 and HSV-2 stimulation, respectively; Table 2) than in the control group (40 and 37 SFC for HSV-1 and HSV-2 stimulation; $p<0.01$ and $p<0.05$, respectively). On the contrary, HSV-specific T cell responses in patients with low frequency of GH recurrences ( 65 and 65 SFC for HSV-1 and HSV-2 stimulation, respectively) did not significantly differ from that of the control group. The graphical comparison of SFC values in Figure 1 documents the differences among the 
patient groups and the control group. Moreover, the figure displays a trend in pronounced HSV-specific T cell responses in patients with high frequency of $\mathrm{GH}$ recurrence compared with patients with a low frequency of $\mathrm{GH}$ recurrences.

In contrast, surface expression of activation markers on circulating CD8+ $\mathrm{T}$ cells did not differ among the study groups. As shown in Table 2, the lack of difference was observed for the percentages of CD38+CD8+ $\mathrm{T}$ cells in patients with high frequency of $\mathrm{GH}$ recurrences, low frequency of $\mathrm{GH}$ recurrences, and the control group $(8.0 \%$, $9.3 \%$ and $9.1 \%$, respectively). Similarly, the percentages of HLA-DR+CD8+ T cells did not differ among patients with high frequency of $\mathrm{GH}$ recurrences, low frequency of $\mathrm{GH}$ recurrences, and the control group $(6.3 \%, 2.6 \%$ and $7.0 \%$, respectively). This suggests that the expression of markers of chronic activation on circulating immune cells is not associated with $\mathrm{GH}$ reactivations.

\section{Discussion}

In this study, we evaluated HSV-specific T cell responses and the expression of immune activation markers on peripheral CD8+ T cells in blood from patients with recurrent GH. The IFN- $\gamma$ Elispot assay was used because it has high sensitivity for the detection of specific $\mathrm{T}$ cells and is simple and reproducible [10]. We observed a positive correlation of HSVspecific T cells with frequency of GH recurrences; however, the recurrences were not associated with changes in expression of activation markers on peripheral CD8+ T cells.

It is well known that virus-specific systemic immune responses are not always effective in the suppression of viral infection. Moreover, the development of chronic infection is frequently accompanied by specific and nonspecific immune activation. A good example is human immunodeficiency virus (HIV) infection, which is associated with strong systemic $\mathrm{T}$ cell responses despite the disease progression $[11,12]$. Similar to HIV, we observed increased levels of HSV-specific $\mathrm{T}$ cells in the blood from patients with high frequency of $\mathrm{GH}$ recurrences. However, in contrast to HIV infection, the signs of systemic immune activation (i.e., elevated expression of CD38 and HLA-DR molecules on CD8+ T cells) were not present in patients with recurrent GH. This discrepancy may be explained by different characters of HIV and HSV infections: HIV infection has a generalized course and is accompanied by peripheral $\mathrm{T}$ cell activation, not only due to massive immune stimulation by high levels of the virus antigens in peripheral blood but also because of their immunostimulatory properties [13]. On the other hand, in GH, HSV infection is mostly localized in the immuno-privileged genital tract and elicits local immune response with minimal impact on the activation of immune cells in the periphery [14]. A suppressive effect of HSV-2 on systemic immune activation was observed in patients with HSV-2 and HIV co-infection [7]. High levels of HSV-specific $T$ cells in the peripheral blood of the patients with high frequency of GH recurrences in our study may be associated with repeated exposure to viral antigens. Similar results were presented by Franzen-Röhl et al. in a prospective study of HSV-2 recurrence after primary infection [8]. Additionally, in the extensive prospective study conducted by Moss et al. [15], the authors did not find a correlation between the frequency of viral shedding or frequency of $\mathrm{GH}$ recurrences and the magnitude of HSV-specific CD4+ T cell response. In our assay, we measured total T cell response to HSV antigens, including not only CD4+ but also CD8+ T cells, that were previously identified as a major component of cellular immune response to herpes virus infection [16]. Nevertheless, our data may support the view that the rate of HSVspecific peripheral $\mathrm{T}$ cell response reflects repeated antigenic stimulation rather than the level of immunosurveillance of the virus latency. The latter seems to be provided mainly by HSV-specific CD8+ $\mathrm{T}$ cells localized in latently infected ganglia [4,17].

We observed that the specific $\mathrm{T}$ cell responses induced by HSV-1 and HSV-2 native antigens were similar even though the vast majority of patients had the recurrent $\mathrm{GH}$ caused by HSV-2. Moreover, the $\mathrm{T}$ cell responses after stimulation with HSV-1 native antigens in seven patients with GH with anti-HSV-1 negative serology did not differ from the HSV-1 seropositive study subjects (data not shown). These findings can be explained by the cross-reactivity of HSV-1 and HSV-2 viral antigens containing both the type-specific and type-common antigenic determinants. Alternatively, some HSV-infected individuals may mount a specific cellular immune response in the absence of detectable HSV-specific antibodies [18]. On the other hand, neither the magnitude of HSV-2-specific cellular immune responses nor the titres of specific antibody were associated with the acquisition of HSV-2 infection by vaccinated individuals [19]. Taken together, these findings support the notion that, at present, there are no available correlates of protective immunity against either primary or recurrent HSV-2 infection [20].

Our study has several limitations. First, the stimulation of T cells with HSV-1 or HSV-2 native antigens is not an optimal approach because both viruses share common antigenic determinants [21]. Moreover, it was shown that native viral antigens ex vivo stimulate mostly CD4+ T cells, while CD8+ T cell stimulation is not very effective [22]. On the other hand, the use of HSV native antigens has some advantages over the use of specific viral peptides because it eliminates differences in immunodominance among different peptides, does not depend on HLA specificity, and contains many adjuvant Toll-like receptor ligands that increase the effectiveness of antigen presentation [23]. Second, the diagnosis of HSV-2 infection was confirmed by the detection of HSV DNA in genital ulceration only in 18 of 26 enrolled subjects. The remaining eight patients had a diagnosis of recurrent $\mathrm{GH}$ of HSV-2 etiology established based on characteristic clinical findings of recurrent genital ulcerations responding to oral acyclovir therapy and positive HSV-2 serology. Thus, we cannot exclude the possibility that some cases of recurrent GH were caused by HSV-1. However, the number of GH recurrences in these patients was relatively high, which supports HSV-2 as the cause of recurrent $\mathrm{GH}$ because $\mathrm{HSV}-1$ genital infection is rarely associated with the recurrent disease [24,25]. 


\section{Conclusion}

These findings indicate that high numbers of systemic HSVspecific T cells are not a marker of immune control of latent genital HSV infection. However, the immune responses in the genital compartment can be totally different. Therefore, studies aimed at HSV-2-specific immunity in the genital tract are warranted, because better understanding of immune mechanisms leading to suppression $\mathrm{GH}$ recurrences is necessary for successful development of efficient therapeutic vaccines.

\section{Data Availability}

Requests for data, 12 months after initial publication, will be considered by the corresponding author.

\section{Additional Points}

Key Messages. (i) The high frequency of genital herpes recurrences positively correlates with the enhanced ex vivo T cell responses to HSV -1 and HSV -2 native antigens. (ii) $\mathrm{T}$ cells from patients with recurrent HSV-2 genital herpes react to HSV-1 and HSV-2 native antigens with similar frequencies. (iii) Recurrent genital herpes is not associated with increased activation of peripheral blood CD8+ T cells.

\section{Conflicts of Interest}

The authors declare no conflicts of interest.

\section{Authors' Contributions}

$\mathrm{MH}$ and $\mathrm{OB}$ were involved in the design and conducting of the study and collaborated in the writing of the manuscript. AS, PCH, and SA were also involved in the conduction of the study. AS participated in the data analysis and writing of the manuscript. KR participated in the interpretation of results.

\section{Acknowledgments}

The study was financed by the institutional support of Charles University.

\section{References}

[1] A. Wald, J. Zeh, S. Selke et al., "Reactivation of genital herpes simplex virus type 2 infection in asymptomatic seropositive persons," New England Journal of Medicine, vol. 342, no. 12, pp. 844-850, 2000.

[2] J. Rajcáni and V. Durmanová, "Early expression of herpes simplex virus (HSV) proteins and reactivation of latent infection," Folia Microbiologica, vol. 45, no. 1, pp. 7-28, 2000.

[3] J. Zhu, D. M. Koelle, J. Cao et al., "Virus-specific CD8+ T cells accumulate near sensory nerve endings in genital skin during subclinical HSV-2 reactivation," The Journal of Experimental Medicine, vol. 204, no. 3, pp. 595-603, 2007.

[4] K. M. Khanna, R. H. Bonneau, P. R. Kinchington, and R. L. Hendricks, "Herpes simplex virus-specific memory CD8+ T cells are selectively activated and retained in latently infected sensory ganglia," Immunity, vol. 18, no. 5, pp. 593603, 2003.
[5] D. M. Koelle, L. Corey, R. L. Burke et al., "Antigenic specificities of human CD4+ T-cell clones recovered from recurrent genital herpes simplex virus type 2 lesions," Journal of Virology, vol. 68, no. 5, pp. 2803-2810, 1994.

[6] E. Franzen-Röhl, D. SchepisM. Lagrelius et al., "Increased cellmediated immune responses in patients with recurrent herpes simplex virus type 2 meningitis," Clinical and Vaccine Immunology, vol. 18, no. 4, pp. 655-660, 2011.

[7] P. M. Sheth, S. Sunderji, L. Y. Y. Shin et al., "Coinfection with herpes simplex virus type 2 is associated with reduced HIVspecific T cell responses and systemic immune activation," The Journal of Infectious Diseases, vol. 197, no. 10, pp. 1394-1401, 2008.

[8] E. Franzen-Röhl, D. Schepis, F. Atterfelt et al., "Herpes simplex virus specific $\mathrm{T}$ cell response in a cohort with primary genital infection correlates inversely with frequency of subsequent recurrences," Sexually Transmitted Infections, vol. 93, no. 3, pp. 169-174, 2017.

[9] Z. Bartovska, O. Beran, H. Rozsypal, and M. Holub, "Antiretroviral treatment of HIV infection does not influence HIV specific immunity but has an impact on non-specific immune activation," Current HIV Research, vol. 9, no. 2, pp. 88-94, 2011.

[10] S. A. Calarota and F. Baldanti, "Enumeration and characterization of human memory $\mathrm{T}$ cells by enzyme-linked immunospot assays," Clinical and Developmental Immunology, vol. 2013, Article ID 637649, 8 pages, 2013.

[11] M. M. Addo, X. G. Yu, A. Rathod et al., "Comprehensive epitope analysis of human immunodeficiency virus type 1 (HIV-1)-specific T-cell responses directed against the entire expressed HIV-1 genome demonstrate broadly directed responses, but no correlation to viral load," Journal of Virology, vol. 77, no. 3, pp. 2081-2092, 2003.

[12] O. Beran, M. Holub, J. Špála et al., "CD38 expression on CD8+ $\mathrm{T}$ cells in Human immunodeficiency virus 1-positive adults treated with HAART," Acta Virologica, vol. 47, no. 2, pp. 121-124, 2003.

[13] F. Nicoli, V. Finessi, M. Sicurella et al., "The HIV-1 Tat protein induces the activation of CD8+ T cells and affects in vivo the magnitude and kinetics of antiviral responses," PLoS One, vol. 8, no. 11, Article ID e77746, 2013.

[14] H. Shin and A. Iwasaki, "Generating protective immunity against genital herpes," Trends in Immunology, vol. 34, no. 10, pp. 487-494, 2013.

[15] N. J. Moss, A. Magaret, K. J. Laing et al., "Peripheral blood CD4 T-cell and plasmacytoid dendritic cell (pDC) reactivity to herpes simplex virus 2 and pDC number do not correlate with the clinical or virologic severity of recurrent genital herpes," Journal of Virology, vol. 86, no. 18, pp. 9952-9963, 2012.

[16] N. Torti and A. Oxenius, "T cell memory in the context of persistent herpes viral infections," Viruses, vol. 4, no. 7, pp. 1116-1143, 2012.

[17] B. S. Sheridan, J. E. Knickelbein, and R. L. Hendricks, “CD8 $\mathrm{T}$ cells and latent herpes simplex virus type 1: keeping the peace in sensory ganglia," Expert Opinion on Biological Therapy, vol. 7, no. 9, pp. 1323-1331, 2007.

[18] C. M. Posavad, M. Remington, D. E. Mueller et al., "Detailed characterization of $\mathrm{T}$ cell responses to herpes simplex virus-2 in immune seronegative persons," The Journal of Immunology, vol. 184, no. 6, pp. 3250-3259, 2010.

[19] R. B. Belshe, P. A. Leone, D. I. Bernstein et al., "Efficacy results of a trial of a herpes simplex vaccine," New England Journal of Medicine, vol. 366, no. 1, pp. 34-43, 2012. 
[20] A. Rompalo, "Preventing sexually transmitted infections: back to basics," Journal of Clinical Investigation, vol. 121, no. 12, pp. 4580-4583, 2011.

[21] D. K. Braun, L. Pereira, B. Norrild, and B. Roizman, “Application of denatured, electrophoretically separated, and immobilized lysates of herpes simplex virus-infected cells for detection of monoclonal antibodies and for studies of the properties of viral proteins," Journal of Virology, vol. 46, no. 1, pp. 103-112, 1983.

[22] H. T. Maecker, S. A. Ghanekar, M. A. Suni, X.-S. He, L. J. Picker, and V. C. Maino, "Factors affecting the efficiency of CD8+ T cell cross-priming with exogenous antigens," The Journal of Immunology, vol. 166, no. 12, pp. 7268-7275, 2001.

[23] R. A. Damhof, J. W. Drijfhout, A. J. Scheffer, J. B. Wilterdink, G. W. Welling, and S. Welling-Wester, "T cell responses to synthetic peptides of herpes simplex virus type 1 glycoprotein $\mathrm{D}$ in naturally infected individuals," Archives of Virology, vol. 130, no. 1-2, pp. 187-193, 1993.

[24] L. Solomon, "Epidemiology of recurrent genital herpes simplex virus types 1 and 2," Sexually Transmitted Infections, vol. 79, no. 6, pp. 456-459, 2003.

[25] R. Patel, O. J. Kennedy, E. Clarke et al., "2017 European guidelines for the management of genital herpes," International Journal of STD \& AIDS, vol. 28, no. 14, pp. 1366-1379, 2017. 